comparison with the $\mathrm{HC}$. The latter PSD analysis resulted positively correlated with ESR $(p=0.01 ; r=0.5)$ and CRP values $(p=0.001 ; r=0.4)$. FP mean differential sensitivity and FP mean defect values were lower in PsA patients with respect to $\mathrm{HC}(\mathrm{p}<0.0001$ for both the comparisons, figure $1 \mathrm{E}-\mathrm{F})$ and resulted negatively correlated with the age $(p=0.03 r=-0.4$ for both the correlations, figure $1 \mathrm{G}-\mathrm{H})$. SDOCT in the posterior pole (superior and inferior hemifields) did not reveal differences for the mean retinal thickness between PsA patients and $\mathrm{HC}$
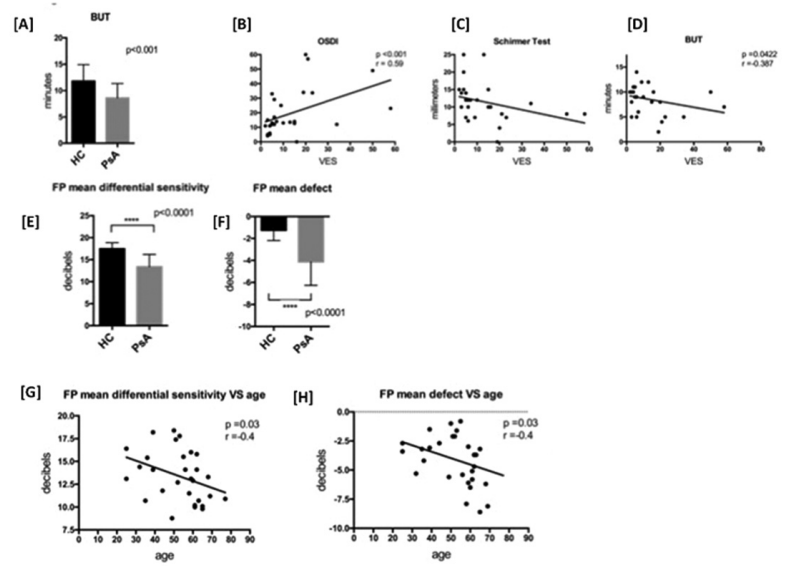

Conclusions: Intriguingly, an impairment in quality of tear film in PsA patients was observed compared to HC. The correlation between ESR and dry eye tests may be explained with a potential relationship between systemic inflammation and sicca syndrome.

Interestingly, PsA patients showed a retinal functional impairment by reduced retinal sensitivity measured by MD, FP mean differential sensitivity and FP mean defect values.

To our knowledge this is the first study investigating eye function and morphology in PsA patients. Further studies are needed to confirm and explain these results. Disclosure of Interest: None declared

DOI: 10.1136/annrheumdis-2018-eular.4554

\section{SAT0309 UNDERESTIMATION OF CARDIOVASCULAR EVENTS BY CARDIOVASCULAR RISK SCORES IN PSORIATIC ARTHRITIS PATIENTS}

H.M. Lam ${ }^{1}$, S.H.O. Ngai ${ }^{1}$, S.H. Cho ${ }^{1}$, T.K. Chun ${ }^{1}$, K.L. Kot ${ }^{1}$, C.T. Yim ${ }^{1}$, E.F. Yu ${ }^{1}$, E. W.L. Kun ${ }^{2}$, L.S. Tam ${ }^{1} .{ }^{1}$ Department of Medicine and Therapeutics, THE CHINESE UNIVERSITY OF HONG KONG, Sha Tin; ${ }^{2}$ Department of Medicine, Alice Ho Miu Ling Nethersole Hospital, Tai Po, Hong Kong

Background: Compared with the general population, patients with Psoriatic Arthritis (PsA) have elevated risks of developing cardiovascular diseases (CVD). The performances of established CVD risk scores in PsA patients have not been fully evaluated yet. European League Against Rheumatism (EULAR) recommends a 1.5 multiplication factor to these CVD risk scores when it is applied in rheumatoid arthritis patients. Whether the same multiplication factor could improve the performance of the risk scores in PsA patients is unknown.

Objectives: To investigate the performances of various CVD risk scores and their EULAR modified versions for predicting CVD events in PsA patients

Methods: Prospectively collected data from the two Hong Kong PsA cohort was used. Discriminatory ability for CV risk prediction was estimated by the area under the receiver operating characteristic curve (AUC). Four different CVD risk scores namely Framingham risk score (FRS), QRISK II, HeartScore and American College of Cardiology and American Heart Association (ACC/AHA) 10 year atherosclerotic cardiovascular disease (ASCVD) and their EULAR modified versions were calculated at baseline. The primary outcome was first CVD events, including stable and unstable angina, myocardial infarction, ischaemic and haemorrhagic stroke, transient ischaemic attack, heart failure, coronary insufficiency, pericardial disease, peripheral arterial disease, thrombosis, percutaneous coronary intervention (angioplasty), coronary artery bypass graft, implantation of pacemaker or defibrillator and CVD death.

Results: 228 patients [48.9 \pm 11.8 years, male: $124(54.4 \%)]$ were recruited between 2006 to 2016. Baseline data were available from 227, 226, 226 and 188 patients to calculate the FRS, QRISKII, HeartScore and ASCVD, respectively. After a mean follow up of $6.7 \pm 4.7$ years, 30 patients $(13.2 \%)$ experienced a CVD event (CVD +group). At baseline, the CVD + group was significantly older $(57.8$ \pm 12.0 vs $47.6 \pm 11.2$ years; $p<0.001)$, with a higher prevalence of diabetes $(26 \%$ vs $12 \% ; p<0.021$ ), had higher systolic blood pressure (SBP: $142 \pm 22.0$ vs 128 $\pm 19.6 \mathrm{mmHg} ; \mathrm{p}<0.001$ ) and higher triglycerides (TG: $1.9 \pm 1.3$ vs $1.4 \pm 0.8 \mathrm{mmol} / \mathrm{L}$, $\mathrm{p}=0.027$ ). All CVD risk scores were significantly higher in the CVD +group (FRS: $18.3 \pm 13.1$ vs $8.9 \pm 8.7, p<0.001$; QRISKII: $11.9 \pm 8.6$ vs $4.9 \pm 5.0, p<0.001$; HeartScore: $2.3 \pm 2.1$ vs $0.9 \pm 1.3, p=0.001$; ASCVD: $14.5 \pm 12.8$ vs $4.8 \pm 5.2, p=0.001$ ) AUC for FRS, QRISKII, HeartScore and ASCVD were $0.74(0.64-0.83, p<0.001)$ $0.76(0.66-0.86, \mathrm{p}<0.001), 0.72(0.62-0.83, \mathrm{p}<0.001)$, and $0.77(0.67-0.86$ $\mathrm{p}<0.001)$, respectively. In total, $76(33.5 \%), 9(4.0 \%), 7(3.1 \%)$ and $47(25.0 \%)$ patients were classified as high CVD risk according to FRS $>10 \%$, QRISK $\|>20 \%$, HeartScore $>5 \%$ and ASCVD $>7.5 \%$ respectively. In the CVD +group, those identified as high risk were only $63 \%$ (by FRS), 20\% (by QRISK2), 13.3\% (by HeartScore) and 46\% (by ASCVD) (figure1a). By applying the EULAR multiplication factor, $80 \%, 36 \%, 26.67 \%$ and $56.7 \%$ of the patients with CVD +were reclassified as high risk (figure $1 \mathrm{~b}$ ).

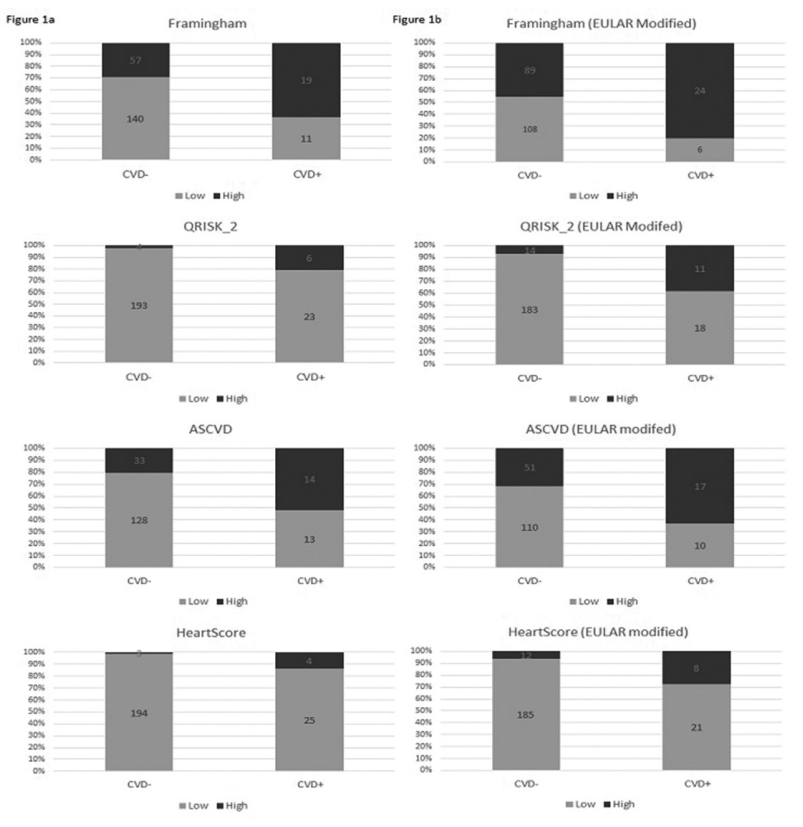

Abstract SAT0309 - Figure 1

Conclusions: All CVD risk scores significantly underestimated CVD risks among PsA patients. This study demonstrated for the first time that adaptation of the EULAR recommendation only improved the accuracy of FRS to a moderate level. Disclosure of Interest: None declared

DOI: 10.1136/annrheumdis-2018-eular.6074

\section{SAT0310 THE ASSOCIATIONS OF SERUM IL18 AND OSTEOPROTEGERIN (OPG) LEVELS WITH THE LIPID PROFILE IN PSORIATIC ARTHRITIS (PSA) PATIENTS}

K. Bonek ${ }^{1}$, P. Gluszko ${ }^{1}$, E. Kontny ${ }^{2} .{ }^{1}$ Department of Rheumatology; ${ }^{2}$ Department of Pathophysiology and Immunology, National Institute of Geriatrics, Rheumatology and Rehabilitation, Warszawa, Poland

Background: We have previously found that IL-18 and OPG serum concentrations are correlated with cardiovascular (CV) risk in psoriatic arthritis but not in ankylosing spondylitis (AS) patients. ${ }^{1}$

Objectives: To investigate whether in PsA patients the association of OPG and IL-18 with CV risk is mediated by an impact of these cytokines on lipid profile changes.

Methods: 49 patients with PsA (25 M/24 F) with $(n=10)$ and without $(n=39)$ coronary heart disease (CHD), and 25 sex and age matched (mean age 44,4 vs 43,4 years) patients with AS were enrolled. Disease activity was measured by DAPSA $(25,17 \pm 19,9)$ in PsA group and by BASDAI $(5,37 \pm 2)$ and ASDAS- CRP $(3,18 \pm 1)$ in AS group. The lipid profile (triglycerides - TG, total cholesterol - tChol, low- and high-density lipoprotein - LDL and HDL, respectively), systemic inflammation markers and cytokines (OPG, IL-18) were measured in patients serum samples Atherogenic index $(\mathrm{Al}=\mathrm{tChol} / \mathrm{HDL})$ was calculated. Statistical analysis was performed using Mann-Whitney U-test and Spearman's Rank test. Data are expressed as mean values.

Results: Patients with PsA presented more atherogenic lipid profile than AS patients because of their higher TG levels (153 vs $126,6 \mathrm{mg} / \mathrm{dl} ; \mathrm{p}=0,05)$ and $\mathrm{Al}$ values $(3,83$ vs 3,$24 ; p=0,05)$ while lower HDL concentrations $(51,6$ vs $61,4 \mathrm{mg} /$ 